\title{
Something better for the weekend?
}

\author{
Peter M.A. Calverley
}

Affiliations: University Hospital Aintree, Clinical Science, Liverpool, UK.

Correspondence: Peter M.A. Calverley, University Hospital Aintree, Clinical Science, Longmoor Lane, Liverpool, L9 7AL, UK. E-mail: pmacalaliverpool.ac.uk

"... and He rested on the seventh day from all His work, which He had made"

Genesis 2:2 (King James' Bible)

The idea of pausing for a day from the normal tasks of life has a long history, possibly began in Babylon where the 28th day of each lunar month was a rest day. Subsequently, this was taken up by the Abrahamic religions, Judaism, Christianity and Islam, which have done much to shape the pattern of the week for billions of people globally. Even Buddhists believe that every seventh or eighth day should be a day of rest, with only the Hindu's not specifying a specific rest day. For centuries, the need to celebrate the Sabbath determined the ebb and flow of life but in the late 19th century, with growing industrialisation in Western Europe and North America, it became possible to take more time away from work. This led to the rise of organised sports on Saturday afternoons in non-Jewish communities and the idea of the weekend, when people who were no longer engaged in "productive" work had time to meet together and pursue other interests.

Of course, these social trends did not affect everyone. For instance, there is no evidence that illnesses are any less serious when they present at the weekend and for those patients who required hospital treatment, care givers were still needed. When medical care focussed mainly on bed rest, regular meals and good nursing there was little need to run a hospital at full capacity 7 days a week. However, times have changed and the burden of acute medicine has moved to patients of greater age afflicted with multiple pathologies. Additionally, both the hours worked and experience of the junior doctors who previously ran the hospital at weekends has decreased. These changes have led to increasing concerns that care for those unlucky enough to be admitted on a Friday may not be as effective as would be the case during the normal working week. The advent of "big data" (databases of sufficient size to control for multiple confounding convariables) has allowed epidemiologists and public health doctors to test the idea that care differs by the day of admission.

In 2001, Bell and ReDelmeier [1] identified a significant increase in mortality in Canadian patients hospitalised with ruptured aortic aneurysm, epiglottitis and pulmonary embolism when admitted at the weekend. Subsequent studies found significant differences in surgical outcomes [2], and in the risk of dying from myocardial infarction and other cardiac conditions where interventional procedures might prove lifesaving [3]. Other studies have shown that these worse outcomes occur with weekend admission in many acute medical conditions, although not necessarily in stoke patients cared for in specialist stoke units [4-6]. Worryingly, the excess mortality at the weekend was at least as great when patients were admitted electively as in those presenting as emergencies [7]. More recently, BARBA et al. [8] noted that mortality among chronic obstructive pulmonary disease (COPD) patients was greater in the $48 \mathrm{~h}$ after admission for an exacerbation when it occurred at the weekend. However, to date, we have much less data about how significant the day of hospitalisation is for our respiratory patients.

@ERSpublications

Death from COPD and pneumonia is more common after weekend hospitalisation; action is needed to address this http://ow.ly/xP4HH

Received: June 042014 | Accepted: June 052014

Conflict of interest: None declared.

Copyright @ERS 2014 
This deficiency has now been remedied by SuISSA et al. [9] who report the results of a characteristically careful and robust analysis of the Quebec Provisional Health Database in this issue of the European Respiratory Journal. They sought to establish whether being admitted in the Friday to Sunday period with a diagnosis of COPD or pneumonia was associated with a greater risk of dying and, additionally, whether this held true for patients who stayed in hospital over these days. Using methods refined in earlier studies [10, 11], they identified 323895 hospitalisations of COPD or pneumonia in patients aged $>50$ years in a 15 -year period from 1990 onwards, approximately two-thirds of which were due to COPD. Subsequently 32414 patients died at a rate of 8.0 deaths per 1000 patients per day. In this study, admission on a Friday did not increase the risk of subsequent death but being admitted to hospital on a Saturday or Sunday did, with an $8 \%$ higher mortality compared with the Monday to Thursday period. Overall, mortality was $5 \%$ higher for patients in hospital on a Friday and 7\% higher if they stayed in on a Saturday or Sunday. A difference that held true irrespective of the total duration of hospitalisation, the presences of other diagnosis, sex or the time of year. Rather worryingly, this pattern showed no change over the 15-year window of observation.

As always, it is possible to create scenarios to mitigate these findings. Perhaps sicker people came into hospital at weekends or patients delayed seeking help until the end of the working week, thus precipitating a crisis, as was suggested after the publication of the original COPD exacerbation data [12]. Appealing as such explanations might be, SuISSA et al. [9] found no evidence to support them. If, as appears likely, overall management of patients is less efficient at the weekends, then this excess mortality may be a marker of the quality of overall management. If this is the case, then we might see more frequent rehospitalisation or worse 30-day outcomes in patients who presented at the weekends and survive, data which would be worth investigating.

For now, the finger is firmly pointed at how we look after our patients throughout the week. There is already an economic argument to use expensive diagnostic facilities over 7 days of work rather than 5 days. Developing a true $24 \mathrm{~h}$ hospital culture remains a real challenge, not least because the European Working Time Directive restricts the amount of time an individual doctor might spend with their patient. Thus a move to a $24 / 7$ working would need to try and integrate the principles of continuity of care better than it is at present. Nonetheless, there are data suggesting that worse outcomes at weekends are associated with low numbers of medical staff on duty and that introducing more senior physicians can decrease the weekend risk $[13,14]$. Clearly we must do something as the present lottery of care based on the day of admission is unacceptable. As pulmonary specialists we should develop appropriate standards of care and contribute to the debate about how and by whom our patients should be managed. If that means more access to specialist pulmonary care earlier in the patients admission then that would not be a bad thing and might help address the problems in Canada that SuIsSA et al. [9] have so clearly highlighted.

References

1 Bell CM, Redelmeier DA. Mortality among patients admitted to hospitals on weekends as compared with weekdays. N Engl J Med 2001; 345: 663-668.

2 Aylin P, Alexandrescu R, Jen MH, et al. Day of week of procedure and 30 day mortality for elective surgery: retrospective analysis of hospital episode statistics. BMJ 2013; 346: f2424.

3 Kostis WJ, Demissie K, Marcella SW, et al. Weekend versus weekday admission and mortality from myocardial infarction. N Engl J Med 2007; 356: 1099-1109.

4 Kazley AS, Hillman DG, Johnston KC, et al. Hospital care for patients experiencing weekend $v s$ weekday stroke: a comparison of quality and aggressiveness of care. Arch Neurol 2010; 67: 39-44.

5 Dorn SD, Shah ND, Berg BP, et al. Effect of weekend hospital admission on gastrointestinal hemorrhage outcomes. Dig Dis Sci 2010; 55: 1658-1666.

6 Sakhuja A, Schold JD, Kumar G, et al. Outcomes of patients receiving maintenance dialysis admitted over weekends. Am J Kidney Dis 2013; 62: 763-770.

7 Mohammed MA, Sidhu KS, Rudge G, et al. Weekend admission to hospital has a higher risk of death in the elective setting than in the emergency setting: a retrospective database study of national health service hospitals in England. BMC Health Serv Res 2012; 12: 87.

8 Barba R, Zapatero A, Losa JE, et al. The impact of weekends on outcome for acute exacerbations of COPD. Eur Respir J 2012; 39: 46-50.

9 Suissa S, Dell'Aniello S, Suissa D, et al. Friday and weekend hospital stays: effects on mortality. Eur Respir J 2014; 44: 627-633.

10 Suissa S, Patenaude V, Lapi F, et al. Inhaled corticosteroids in COPD and the risk of serious pneumonia. Thorax 2013; 68: 1029-1036.

11 Suissa S, Dell'Aniello S, Ernst P. Long-term natural history of chronic obstructive pulmonary disease: severe exacerbations and mortality. Thorax 2012; 67: 957-963.

12 van den Borst B, Wesseling G. Acute exacerbations of COPD: it's the weekend but it can't wait until Monday. Eur Respir J 2012; 39: 1547.

13 Bell D, Lambourne A, Percival F, et al. Consultant input in acute medical admissions and patient outcomes in hospitals in England: a multivariate analysis. PloS One 2013; 8: e61476.

14 Brims FJ, Asiimwe A, Andrews NP, et al. Weekend admission and mortality from acute exacerbations of chronic obstructive pulmonary disease in winter. Clin Med 2011; 11: 334-339. 